\title{
Systematic Analysis of a New Emergency Medicine Ultrasound Rotation
}

\begin{abstract}
Introduction: Emergency Ultrasound (EUS) is a vital tool for the diagnosis and management of emergency department patients. EUS training is mandated by the Accreditation Council for Graduate Medical Education (ACGME) for all Emergency Medicine (EM) residencies and each program is required to assess resident competence in EUS. We sought to determine the competence of EM residents at a new EM residency training center by evaluating technical accuracy after initial EUS training.
\end{abstract}

Methods: We conducted a retrospective review of all ultrasound images saved before and after the each EM resident's EUS rotation. Ultrasound images were evaluated for several imaging components (depth, gain, application type, measurements, adequate views) and rated for technical accuracy.

Results: During the study period, 18 EM residents completed the introductory course and the two week first-year EUS rotation. A total of 1126 EUS exams were performed. 849 educational EUS exams were performed during resident training for educational purposes, and 211 clinical EUS exams were saved during clinical evaluation of ED patients. Of the clinical EUS exams performed prior to the rotation, 42 (62\%; $95 \%$ Confidence interval [Cl] $51 \%$ to $73 \%$ ) were rated as excellent quality, 9 (13\%; $95 \% \mathrm{Cl} 5$ to $21 \%$ ) were rated as good, 5 were rated as fair (7.3\%; $95 \% \mathrm{Cl} 1.3$ to $13 \%$ ) and 12 (17.6\%; $95 \% \mathrm{Cl} 8.6$ to $26.6 \%$ ) were rated as poor. Of the clinical EUS exams performed after the 2 week EUS rotation, $103(72 \%, 95 \% \mathrm{Cl} 64$ to $79 \%)$ were rated as excellent, 21 (14.6\%; $95 \% \mathrm{Cl} 8.8$ to $20 \%$ ) were rated as good, 7 (4.8\%; $95 \% \mathrm{Cl} 1.3$ to $8.3 \%$ ) were rated as fair and $12(8.3 \% ; 95 \% \mathrm{Cl} 3.8$ to $12.8 \%)$ were rated as poor.

Discussion: Our findings demonstrate that an introductory course and two-week EUS rotation improved resident technical accuracy with an increase in excellent quality exams and a reduction in poor quality exams post intervention.

\section{Introduction}

Emergency Ultrasound (EUS) is a focused, goal-oriented ultrasound examination performed at the patient's bedside by emergency physicians (EP). EUS is used to rapidly diagnose acute life threatening conditions and guide invasive procedures. EP-performed ultrasound is easily learned, highly accurate, and expedites care by facilitating early diagnosis and decreasing the patient's length of stay [1-4]. Because of the benefit provided to emergency patients, research and training in EUS has grown dramatically over the last two decades.

The first model curriculum for EUS training was published in 1994 [5]. Since then, the use of EUS has become widespread and is increasingly incorporated into routine clinical practice. Recognizing that there was variability in residency training in EUS [6-8], the Council of Residency Directors (CORD) presented recommendations, which delineate the minimum standards for EUS education for emergency medicine (EM) residents $[9,10]$. Today, EUS

\section{Emergency Medicine \& Critical Care}

\section{Adeyinka Adedipe*, Brandon Backlund, Kari} Rood, Dana Sajed and Sachita Shah

Division of Emergency Medicine, Department of Medicine, University of Washington School of Medicine, United States of America

\section{Address for Correspondence}

Adeyinka A Adedipe, MD, RDMS, Division of Emergency Medicine, Department of Medicine, University of Washington School of Medicine, University of Washington Medical Center,1959 NE PACIFIC STREET, BOX 356123, SEATTLE, WA, United States of America 98195-6123, Phone: 206.598.4461; Fax: 206.598.4569; E-mail: adeyinka@uw.edu

Submission: 02 July 2013

Accepted: 20 September 2013

Published: 23 September 2013

is one of three core procedures assessed by the Accreditation Council for Graduate Medical Education (ACGME) and Residency Review Committee (RRC) for Emergency Medicine during accreditation visits. Additionally, the RRC requires each program to assess resident performance and competency in EUS.

While no standard currently exists for EUS training in residency, successful EM programs provide a mix of education that includes an introductory course, didactics, a dedicated EUS rotation, and ongoing quality assurance with resident feedback [11-13].

We performed a systematic analysis of a new EUS training program at our institution to assess EM resident competency after the first half of their emergency ultrasound rotation. The EUS rotation at the University of Washington begins in the first month of the intern year with an introductory ultrasound workshop. Each EM resident then completes a 2 week block of the EUS rotation during their first year. All residents complete a second 2 week block in the third year of residency, with teaching directed to more advanced EUS concepts and applications. We reviewed all EUS exams performed by EM residents before and after the first 2 week block of the EUS rotation to determine the types of EUS scans recorded, and to assess the technical adequacy of each scan. Additionally, we reviewed EM resident evaluations of the EUS rotation, quarterly workshops, and didactic sessions, to identify potential areas of additional educational need.

\section{Methods}

\section{Study Design}

We conducted a retrospective review of all EUS exams saved in our image database performed by first year EM residents from an academic emergency medicine program in a large urban area in the Pacific Northwest. EUS exams performed during the EUS rotations were categorized as educational EUS exams; EUS exams performed in the evaluation and management of ED patients were categorized as clinical EUS exams.

\section{EUS Quality Assurance}

Each ultrasound examination was reviewed for technical accuracy by an ultrasound fellowship trained emergency physician. Five different components of technical accuracy were evaluated: 
image depth (D), image gain $(\mathrm{G})$, application settings (A), accuracy of caliper measurements (M), and adequate number of images (I). EUS exams were rated as "excellent" if there were no component errors and superior image quality, and "good" if there was a single component error. Exams rated as "fair" had 2 component errors, and "poor" studies had 3 or more component errors. EUS studies with no identifiable anatomic landmarks were rated as "un-interpretable" no matter how many component errors were present.

\section{Emergency Ultrasound Curriculum}

All EM residents participate in the University of Washington EUS training program which begins during the first month of intern year. An introductory skills day (4 hours) includes didactics on physics, focused assessment with sonography for trauma (FAST), focused echocardiography, and early obstetrics ultrasound. EUS faculty proctor EM residents individually at training stations after each lecture. Each training station incorporates live models, healthy volunteers or simulators where appropriate.

Throughout the remainder of the academic year, EUS faculty provides monthly ultrasound didactics and quarterly skills labs dedicated to a single EUS application.

In the PGY1 year, residents have a two week EUS rotation where they are individually proctored by EUS faculty using live models in the emergency department over a broad emergency ultrasound curriculum (see appendix). During the rotation, resident physicians are instructed on proper ultrasound technique and are given immediate feedback. At the end of each rotation, quarterly skills lab and didactic session, EM residents are asked to complete a survey evaluating their experience.

\section{Results}

From July 2011 to June 2013, a total of 1126 EUS exams were available for review. 849 exams were educational EUS exams performed during the 2 week rotation. 280 EUS exams were performed during clinical evaluation of ED patients, of which 211 were saved to the database and available for review (68 performed before the EUS rotation, and 143 after the rotation). During the study period, $18 \mathrm{EM}$ residents completed the introductory course and first year EUS rotation.

Each EM resident performed an average of 47 (range 3364) educational EUS examinations over the course of the 2 week EUS rotation. The most commonly performed educational EUS examinations were FAST (28\%; 95\% Confidence interval [CI] 25\% to $31 \%$ ), focused echocardiography (26\%; 95\% CI 23.1 to $28.9 \%$ ), right upper quadrant (11\%; $95 \%$ CI 8.9 to $13 \%)$, and aorta (10\%; $95 \%$ CI 8 to $12 \%$ ) (Figure 1). EM residents performed and saved a total of 211 clinical EUS exams in the image database and available for review. 68 clinical EUS exams were performed prior to the EUS rotation, and 143 were performed after the EUS rotation.

Of the clinical EUS exams performed prior to the rotation, 42 (61.7\%; $95 \%$ CI 51 to $73 \%$ ) were rated as excellent, 9 (13\%; $95 \%$ CI 5 to $21 \%$ ) were rated as good, 5 ( $7.3 \%$; $95 \%$ CI 1.3 to $13 \%)$ fair and $12(17.6 \%$; $95 \%$ CI 8.6 to $26.6 \%)$ were rated as poor. Of the 12 clinical EUS exams rated as poor, all had inadequate views and 8 (66\%) had inadequate depth. Focused OB ultrasound exams were the most frequently occurring EUS exam rated as poor (33\% of total poor quality studies). Of the ultrasound exams performed after the

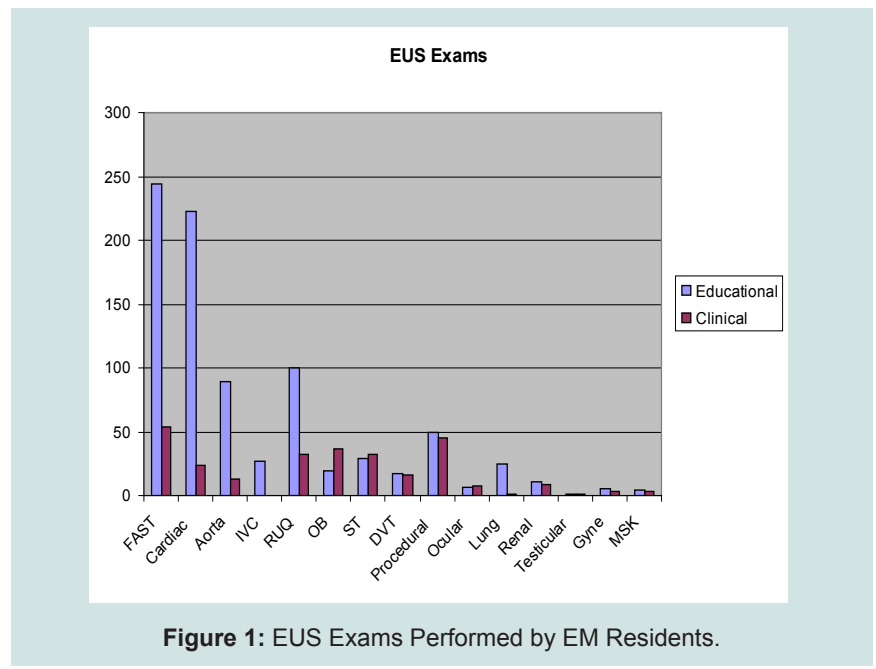

2 week EUS rotation, 103 (72\%; 95\% Confidence interval [CI] 64\% to $79 \%)$ were rated as excellent, 21 (14.6\%; $95 \%$ CI 8.8 to $20 \%)$ were rated as good, 7 ( $4.8 \%$; 95\% CI 1.3 to $8.3 \%)$ were rated as fair, and 12 (8.3\%; $95 \%$ CI 3.8 to $12.8 \%$ ) were rated as poor (Figure 2). Of the EUS exams rated as poor, 10 had incomplete views and 7 had poor image gain, and inaccurate measurements. The exam type most commonly associated with technical errors was OB (7/12 or 58\%). In addition, one EUS exam in each of FAST, renal, RUQ, aorta and pelvis/gyne were rated poorly.

Most EUS exams performed by an EM resident after the 2 week rotation demonstrated a decrease in average number of component errors (Figure 3). Specifically FAST (56\%), focused echocardiography $(65 \%)$ and renal US (70\%) demonstrated modest decrease in average component errors. Aorta (40\%) and OB ultrasound (22\%) had a notable increased number of component errors when performed after the 2 week rotation.

Over the study period 17 lectures were given along with six different skills workshops. After each didactic session and workshop, a survey was distributed to the attendees, and aggregate data from these surveys were used for this analysis. A total of 18 responses were collected for the lecture-based EUS educational content and for the EUS workshops. Overall, the EUS workshops received higher overall scores compared to the didactics ( 9.73 vs. 8.85 out of 10 ). EM resident overall evaluations of the workshops were highest with respect to "appropriateness of didactic content for the practice of Emergency Medicine' and knowledge of presenters. Didactics received the lowest evaluations on citation of current literature and "usefulness of audiovisual aids to the presentation". Aggregate evaluation of the EUS rotation demonstrated an overall rating of 9.96/10.

\section{Limitations}

Our analysis was limited by the unavailability of all clinical EUS exams. 69 clinical EUS exams were not saved and thus could not be reviewed for technical accuracy. This could have effected the overall technical accuracy measurements. In addition, the number of responses to the surveys sent out after the didactic and practical EUS educational modules was low. The opinions of those who did not respond may therefore differ in either a more positive or more negative direction from those who did respond. 


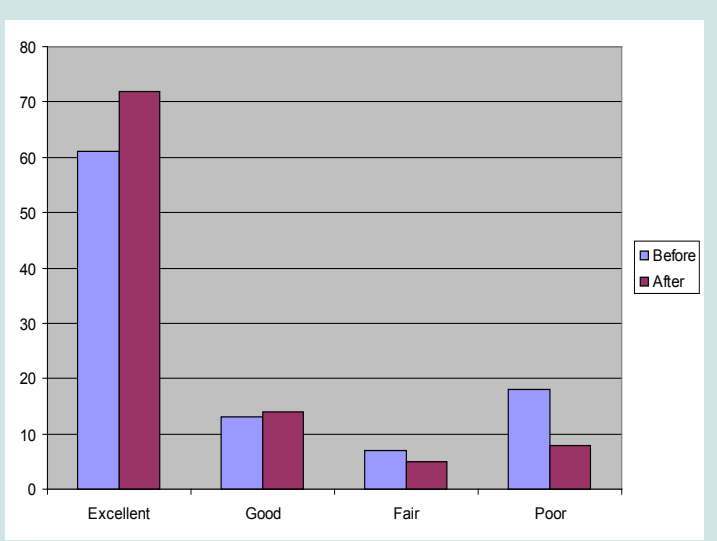

Figure 2: Technical Accuracy Before and After EUS Rotation (Percentages).

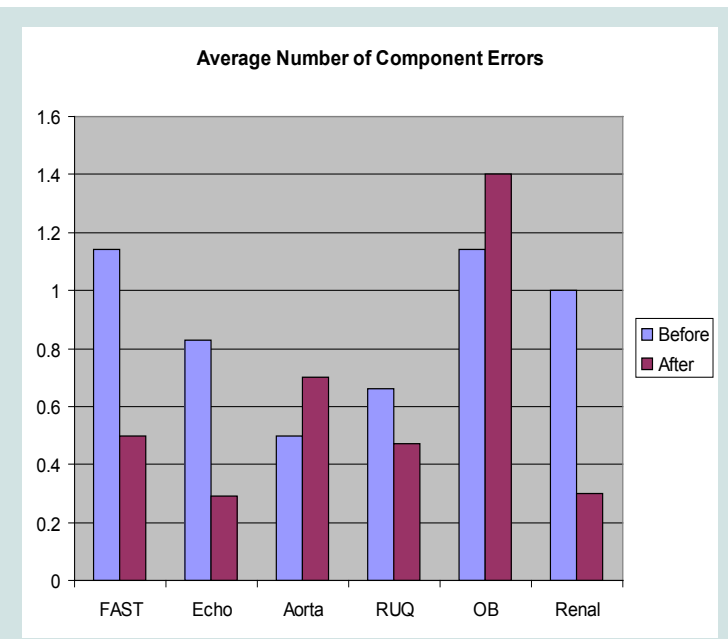

Figure 3: Average Number of Component Errors by Exam Type.

\section{Discussion}

Ultrasound education is required for emergency medicine residencies in the United States. Although no standard currently exists for EUS training in post-graduate education, established programs have dedicated rotations with proctored exams during the rotation. We present our initial assessment of EM resident technical accuracy in performing bedside ultrasound after the completion the first half of a newly developed EUS rotation.

The types of EUS exams performed by these EM residents reflected the scope of practice delineated by ACEP Section of Ultrasound. The most commonly performed exams were FAST, focused echocardiography, RUQ and aorta. The diversity of EUS exams is similar to those performed in the clinical environment. Soft tissue EUS exams were noted to be infrequently performed during the EUS rotation, but frequently performed during clinical rotations. We found that OB EUS examinations were infrequently performed during both the EUS rotation and clinical rotations. We suspect that the low number of OB exams performed accounts for our resident's comparatively low scores for this exam type.

We found that the technical accuracy of EUS exams improved after the first 2 weeks of the EUS rotation. While there was no substantial change in the number of exams rated as good or fair, the percentage of clinical EUS exams rated to be of poor quality decreased by $44 \%$ after the EUS rotation. The number of EUS exams rated as excellent increased by $18 \%$.

Common technical errors associated with EUS exams rated as poor ( 3 or more component errors) were the following: number of views $91 \%$, depth $65 \%$, measurement $60 \%$, application type $47 \%$, and gain $43 \%$. Poor quality EUS exams most commonly had a combination of depth, measurement, and adequate number of views contributing to the technical error. The most common EUS study type associated with poor overall technical accuracy is OB. We suspect that more exposure to obstetric patients during the EUS rotation would have improved the overall technical accuracy of OB exams. Ensuring that EUS rotators have the opportunity to scan OB patients in outpatient clinics, in labor and delivery, or through simulation, could improve outcomes.

Overall, EM residents found the EUS rotation to be beneficial to their EM training experience. Although the number of survey respondents was low, they all agreed that the rotation "helped [them] in becoming an excellent physician" and "added to [their] understanding of the specialty". Some noted uncertainty in regards to having adequate opportunity to meet the objectives of the rotation.

Our results will help guide improvements of our EUS curriculum and may serve as guidelines for new programs at other institutions. We will, pay specific attention to factors associated with poor quality EUS exams, such as delineating the proper number of views and appropriate depth for specific EUS exam types. Additionally, the patient population in our institution limits the exposure of EM residents to $\mathrm{OB}$ patients. We will address this potential knowledge gap with supplemental $\mathrm{OB}$ ultrasound training in order to prepare our residents for their future careers in other healthcare centers.

\section{References}

1. Blaivas M, Harwood RA, Lambert MJ (1999) Decreasing length of stay with emergency ultrasound examination of the gallbladder. Acad Emerg Med 6: 1020-1023.

2. Blaivas M, Sierzenski P, Plecque D, Lambert M (2000) Do emergency physicians save time when locating a live intrauterine pregnancy with bedside ultrasonography? Acad Emerg Med 7: 988-993.

3. Jang TB, Ruggeri W, Dyne P, Kaji AH (2010) Learning curve of emergency physicians using emergency bedside sonography for symptomatic firsttrimester pregnancy. J Ultrasound Med 29: 1423-1428.

4. Jang TB, Ruggeri W, Dyne P, Kaji AH (2010) The learning curve of resident physicians using emergency ultrasonography for cholelithiasis and cholecystitis. Acad Emerg Med 17: 1247-1252.

5. Mateer J, Plummer D, Heller M, Olson D, Jehle D, et al. (1994) Model curriculum for physician training in emergency ultrasonography. Ann Emerg Med 23: 95-102.

6. Ahern M, Mallin MP, Weitzel S, Madsen T, Hunt P (2010) Variability in Ultrasound Education among Emergency Medicine Residencies. West $J$ Emerg Med 11: 314-318.

7. Jang TB, Coates WC, Liu YT (2012) The competency-based mandate for emergency bedside sonography training and a tale of two residency programs. J Ultrasound Med 31: 515-521.

8. Witting MD, Euerle BD, Butler KH (1999) A comparison of emergency medicine ultrasound training with guidelines of the Society for Academic Emergency Medicine. Ann Emerg Med 34: 604-609.

9. Heller MB, Mandavia D, Tayal VS, Cardenas EE, Lambert MJ, et al. (2002) Residency training in emergency ultrasound: fulfilling the mandate. Acad Emerg Med 9: 835-839. 
Citation: Adedipe A, Backlund B, Rood K, Sajed D, Shah S. Systematic Analysis of a New Emergency Medicine Ultrasound Rotation. J Emerg Med Critical Care. 2013;1(1): 4.

ISSN: $2469-4045$

10. Akhtar S, Theodoro D, Gaspari R, Tayal V, Sierzenski P et al. (2009) Resident training in emergency ultrasound: consensus recommendations from the 2008 Council of Emergency Medicine Residency Directors Conference. Acad Emerg Med 16: S32-S6.

11. Costantino TG, Satz WA, Stahmer SA, Dean AJ (2003) Predictors of success in emergency medicine ultrasound education. Acad Emerg Med 10: 180-183.
12. Noble VE, Nelson BP, Sutingco AN, Marill KA, Cranmer H (2007) Assessment of knowledge retention and the value of proctored ultrasound exams after the introduction of an emergency ultrasound curriculum. BMC Med Educ 7: 40.

13. Mahler SA, Swoboda TK, Wang H, Arnold TC (2012) Dedicated emergency department ultrasound rotation improves residents' ultrasound knowledge and interpretation skills. J Emerg Med 43: 129-133. 